\title{
Behaviour and Bioacoustic Characteristic of Male Dolphins Bottle Nose (Tursiops Aduncus) in Captive, Indonesia
}

\author{
Muhammad Zainuddin Lubis* \\ Department of informatics engineering, Indonesia
}

Submission: November 03, 2016; Published: December 12, 2016

*Corresponding author: Muhammad Zainuddin Lubis, Department of informatics engineering, Geomatics engineering, Batam Polytechnic Jalan Ahmad Yani, Batam Centre, Batam 29461, Indonesia Email: zainuddinlubis@polibatam.ac.id; lubiszainuddin@gmail.com

\begin{abstract}
Bioacoustic is knowledge that combines biology and acoustic which usually refer to research on the production of sound, dispersion through an elastic medium , and acceptance in animals, including humans. This research using passive acoustic method and behavior observing of male dolphins by doing approach science of the passive acoustic (bioacoustics) is see the difference patterns and a characteristic sound of the porpoise. Dolphins male (Tursiops aduncus) preserved in park of safari indonesia cisarua bogor, having value intensity lowest of $28.03 \mathrm{~dB}$ and the highest is of $32,01 \mathrm{~dB}$. With an average parameter measurable namely salinity up to $30 \%$ and temperature $23^{\circ} \mathrm{C}$ with the depth of the pool 4,5meters. Range the frequency of sounds click with the highest intensity of 32,01dB with a clicking sound after food in first day to 2 which is found in range frequency namely $14.000-16.000 \mathrm{~Hz}$. Range the frequency of sounds click dolphins male (tursiop aduncus) in park of safari with frequency of $14.221-15.100 \mathrm{~Hz}$. There is a difference intensity voting against treatment before and after eating by using the pool the same study. Value intensity highest obtained at the sound of after food in second day with the intensity $32,01 \mathrm{~dB}$ and value of the lowest intensity of are in before a meal is first day. Dolphins tends to be basic a pool of sea water in as before eating and dolphins always was sitting on the surface of the pond at the time after eating.
\end{abstract}

Keywords: Bioacoustic; Male dolphins bottle nose (Tursiops aduncus); Frequency; Intensity

\section{Introduction}

In the world fisheries own with the methods acoustic passive applied to monitor marine mammals, and biota other on the sea [1]. Signals obtained from recording sound marine mammals very weak that calls for amplification and difficult to determine of which the direction that suarara. Passive is sound derived from animals target [2]. The concept of acoustic passive conducted in marine mammals is to detect a sound when mammals it is in the area measurements in environment scope of the tape recorder. The measurement of is done with use software and also with listen.A method of acoustic passive also used odibidang military service in the development of security system of attackers under water in the estuari by doing recording sound which is raised from diving [3].

Marine mammals hearing in is measured in the subject living with use audiometer behavior or technique elektrofisiologi [4]. For species does not learn from vivo audiometer. With some characteristic of hearing can be is predicted based on the frequency of the results of the production of sound done on the observation the characteristic sounds good is do or not have response behavior in trained animals [5]; or morphological hearing, including the trait of biomechanics from the basilar and characteristic of other sounds [6]. A little bit too known the sound has type yelping (burst), characteristic of spectral, temporal, and amplitude a shrill sound pulse of type the sound of a click very few explored. As for early description in literature that most of it that is worth qualitative, that reflects interpretation hearing subjective and classification hearing in humans [7-10].

The sound of a click generally used for the purpose of echolocation, while the sound of a whistle ledakan-berdenyut and played a major role in internal communication and across group [11]. A whistle that continues indefinitely, giving signals frequency [12], with various wide emissions of $800 \mathrm{~Hz}$ and $28.5 \mathrm{KHz}$ [13] often there are components harmonic [12]. A dolphin start interaction with the provision of signals, with 
information, in tape a given frequency. The signal source then relies on a source of to hear and react to the sound. In hearing to dolphins ranged from about $50 \mathrm{~Hz}-150 \mathrm{KHz}$, with the variation of additional among [12].

For every years, researchers have demonstrated the advantages of hydrophones Single Mode Fiber (SMF) as a potential alternative to the voice navigation and the beginning of the technology/sound navigation and ranging (SONAR). Bioacoustic not be separated from the use of hydrophones as lat voice recorder where the acoustic pressure is recorded on hydrophone is a source of pressure disturbance at sea $(\Delta \mathrm{P})$ relative to the pressure of the ocean in the background on the recording medium depth sea water. Excess sound pressure at sea are usually worth a small $(\sim 10-2 \mathrm{~Pa})$, and it has become the standard in the sea in the literature to show the acoustic sound pressure in the list of the decibel scale $(\mathrm{dB})$ relative to the reference pressure (Po) of $1 \mu \mathrm{Pa}$ [13], In addition, the convention to use $1 \mathrm{~m}$ as a reference distance (ro), namely when calculating the acoustic pressure from the source of the sound.

Bioacoustic is the knowledge that combines biological and acoustics which usually refer to research on the production of sound, dispersion through an elastic medium, and acceptance in animals, including humans. This involves neurophysiology and anatomy for the production and detection sound, as well as the relations of acoustic signals with medium dispersinya. Findings in this area give evidence for us of the evolution of acoustic mechanism, and from there, evolution animals who use them $[14,15]$. But because the science of acoustics grown very much to dolphins, researchers formerly had been exercising records and analysis vocalization [16]. Bioacoustic research is needed to know language communication (acoustic communication) in mammals. Bioacoustic be separated from the use of hydrophone as lat voice recorder where pressures acoustics recorded on hidrofon is the source of the disorder pressure on the $(\Delta \mathrm{P})$ relatively on the background the recording in the medium sea water. This study aims to analyse and distinguishing characteristics the sound of a click dolphins male bottle nose (Tursiops aduncus) in quarantine pool, by using the method bioacoustic and see behavior male dolphins bottle nose (Tursiops aduncus) .

\section{Materials and Methods}

Research activities implemented in March to September 2015. The data activities implemented in safari park Cisarua Bogor, Indonesian. Processing data implemented in laboratory of marine acoustic instrumentation (MAI) in the department of marine science and technology, located in water tank, Bogor Agricultural University.

\section{Tools and Materials}

Tools and material used in the methodology this is hidrofon SQ3, a hg thermometer to measure the temperature of sea water; refractometer for measuring salinity sea water is in swimming; Dolphins EAR 100 hydrophones a serial number DE989505 that is sensors sound underwater camera gopro hero $3+$ serves to record the movement of to dolphins visually. Software MATLAB and WAVELAB that is used to cultivate the data collected. Material used by 2 the tail of a dolphins male bottle nose. The data collected from observation dolphins in the pool research is quarantine pool and do the processing of with using software is WAVELAB and data that had been deposited with WAVELAB software will be conducted filters with cutting the data important that is sound actually the porpoise. After was finished will be conducted data analysis the frequency with a method of bioacoustics by producing fast fourier transform instead (FFT) and extracted with the data .txt and see links with behavior the movement and position of the porpoise is. Analysis next executed is analysis power spectral density (MATLAB). So that characteristic of behavior bottle nose male dolphins can be completed.

\section{Hydrophone}

Hydrophone is an instrument that serves to listen to underwater sound. This tool converts noise coming from inside the sea water into electrical signals, and can then be ampification, analyzed, or played in the air [17]. Hydrophone is usually in the form of a piezo-electric ceramic slab [14]. Standardization of Hydrophone for purposes of biocoustic issued by Bioacoustic Inc. Dolphin ear hydrophones able to detect the frequency of sounds in $1-2 \mathrm{~Hz}$. The threshold lowest hearing of men have earned is only able to obey the voice of to the frequency of $20 \mathrm{~Hz}$. Voices outside the threshold normal hearing man can be heard use dolphin ear hydrophones equipped with WAVELAB software. The form of a device dolphin ear hydrophones can be seen in Figure 1. Specification of dolphin ear hydrophones [18] is range of frequencys: 7-22.0000Hz. Type of tranducer Hydrophone: MPC (Piezo). The form of configuration : Omni Directional. Packed of hydrophone: Rugged epoxy case.

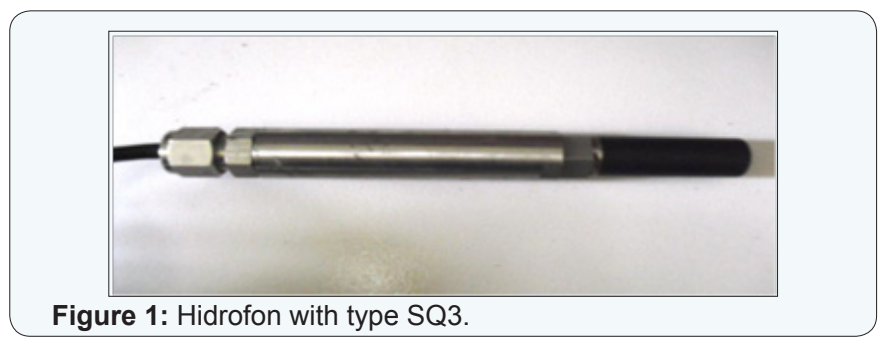

The diameter of $60 \mathrm{~mm}$ and thick $8 \mathrm{~mm}$. Cable type: High Quality, Small noise, with Neoprene/PVC. Size of standart cable: 12Meter. Preamplifer: One packed with line /Earphone output. Power: $\pm 7 \mathrm{~mA}$ to 9 Volt. Limits a range of frequencies provided was the frequency of sounds that can be heard normally by using earphones and used WAVELAB software for recording sound directly from dolphin ear, so a lower limit frequency can be detected will be more low as reach several hz, and the upper limit of a frequency that can be detected can reach more than $22 \mathrm{KHz}$ [19-21]. Hydrophones figure can be seen in Figure 1. 


\section{Fourier transform}

The basis of characteristic frequency on a signal is transformation fourier [22]. Fast fourier transform instead (FFT) is a algorithm to count discrette fourier transform instead (DFT). Functions general of transformation fourier is find components frequency signal that is hidden by a signal the domain time of with noise [23] is :

$$
\begin{aligned}
& S=f f t(y) \ldots . \\
& S=f f t(y, n) . .
\end{aligned}
$$

The form of the orders (1) and (2) almost the same, that is counting dft of vector $x$, only on the order of (2) throne with the use of parameter long FFT (n).

\section{Power Spectral Density}

Power spectral density (PSD) definition as the magnitude of the power per intervals frequency, in the form of mathematics [22]:

$\operatorname{PSD}=\llbracket\left(|\mathrm{Xn}| \rrbracket^{2} / \mathrm{f}\right.$ ([( (Amplitudo $\left.) \rrbracket^{2} / \mathrm{Hz}\right)$.

Calculation psd in MATLAB uses the method welch [23], namely looking for DFT (Based on accounts with algorithms FFT), then squaring the value of the magnitude.

\section{Parameter environment}

Parameter environment measured is temperature and salinity the result of these two physical parameters this is a has the most influence on the level of stress dolphins. Stress experienced therefore, the dolphins can to cause deviations behavior to dolphins. The measurement of temperature sea water in aquariums done by entering a hg thermometer into aquarium. A hg thermometer will be flicts before put into sea water to get any effect muainya and restore the starting point a hg thermometer before measuring started. This sea water temperature obtained by reading on the scale of shown in a hg thermometer.

\section{Processing and analysis data of dolphin sound}

Data processing acoustic using software.The sound produced dolphins bottle nose and yielding fast fourier transform instead (FFT). FFT is algorithm to count transformation fourier discrete (FFT) and its reverse. A transformation fourier change time (or room with the frequency and otherwise. As a result, transformation fourier fast much used for many application in engineering, science, and mathematics [24]. To see to scatter per unit time so with WAVELAB software, and data fft deposited in the form of *.txt. After the process so the data mixed with using software microsoft excel and mixed with using software MATLAB for produce figure. Data recording dolphins sound with interpretation into graphical form the frequency and echo level. A graph produced then analyzed and compared with the visual observation. The resulting then analyzed and compared with the visual observation. The visual observation can be done by see mannerisms of dolphins observed by using the method home video recording a underwater camera when recording of sound dolphins. It is meant to see reaction to dolphins objects or instrument is around by observing mannerisms the porpoises and can see the health of from dolphins during the research/ process of voting. But sketches research male dolphins bottle nose (Tursiops aduncus) in captivity / quarantine pool is presented in Figure 2.

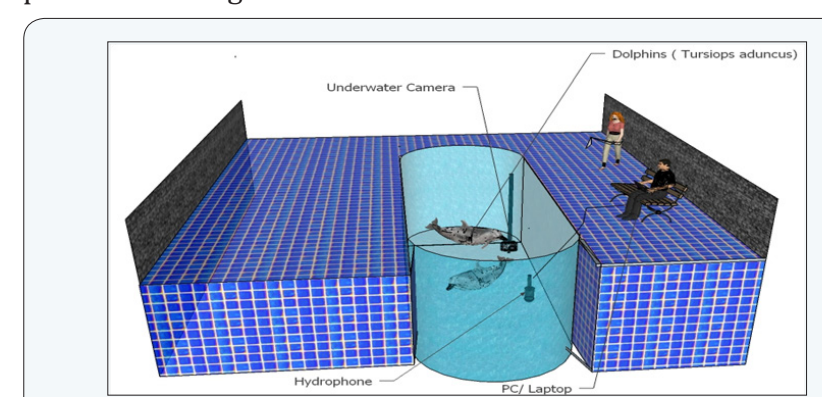

Figure 2: Sketch research male dolphins bottle nose (Tursiops aduncus) in pool of quarantine.

\section{Result and Discussion}

Sound mammal (dolphins) to in this research is the noise that derived from dolphins out of the skull dolphins. According to [25], sound is a very important thing to behavior while communicating to several kinds of dolphins and according to [26,27] mammals can issue various amplitude votes for communicating in exchange information. Information brought from signals sound describing on the state of the danger that threatens, the state of aggressively to scare off our enemies, or call peminangan. A voice too resulting from the impact of behavior such as at eating, move, escape the enemy, and reproduction (sexuality and phase enlargement) [16]. From the data recording sound, dolphins bottle nose (Tursiops truncatus) having three type a different voice.

Third type these sounds can be distinguished only by using normal hearing. Third type the sound was a click, whistle and burst. In the data of sound dolphins done on the and sunday different. The process of recording dolphins sound done three times recording. This research absent from the parameter measurable namely measuring parameter temperature and salinity which is with the pool research that is quarantine pool. Salinity and temperature before and after in the pool quarantine dolphins male to the measurement of parameter are on the day to 1,2 and 3 in the pool quarantine, and time the that is, before and after dolphins eat. The process recording sound of dolphins done in 3 times the parameters of data with a date and sunday different. Researched process will be covered by the parameters of the parameters of measurable is measure of the parameters of temperature and salinity that were on the pool research namely quarantine pool.

Salinity and temperature before and after quarantine pool male dolphins with the measurement of the parameters are in the day to 1, 2 and 3 on the pool quarantine, and time adoption 
of which is before and after eating dolphins with the results of salinity and temperature before eating the magnitude of salinity, which is $30 \%$, while the withdrawal of the data after eating salinity fell to $29 \mathrm{ppm}$ this has led to salinity which is found in quarantine pool already injection with the factors that is somewhere so and the day of second with salinity of $28 \%$ on as before eating and $27 \mathrm{ppm}$ after eating with the temperature before eating $23^{\circ} \mathrm{C}$ and after eating is as much as $22^{\circ} \mathrm{C}$.

\section{Characteristic of Dolphins Sound}

Sound is a very important thing to behavior while communicating to several kinds of fish and according to [28] fish can issue various amplitude votes for communicating in exchange information .Information brought from signals sound describing on the state of the danger that threatens, the state of aggressively to scare off our enemies, or call peminangan. A voice too resulting from the impact of behavior such as at eating, move, escape the enemy, and reproduction (Sexuality and phase enlargement) [16,27]. Result of this research in the form of the sound derived from software WAVELAB was conducted anti logs and mixed with using software MATLAB so as to produce a chart of the sound intensity against frequency. The frequency of a recorded namely $5.200-22.000 \mathrm{~Hz}$. The data shows on a chart namely ping 62 to ping 195 . Spectrum of sound click can be seen in Figure 3, and charts relations frequency to intensity before a meal day to, 1, 2, 3 and after eating day to 2, 3 it can be seen in Figure 4.

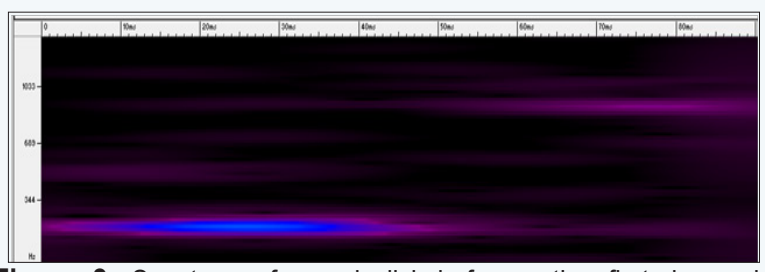

Figure 3: Spectrum of sound click before eating first day male dolphins. (Tursiops aduncus).

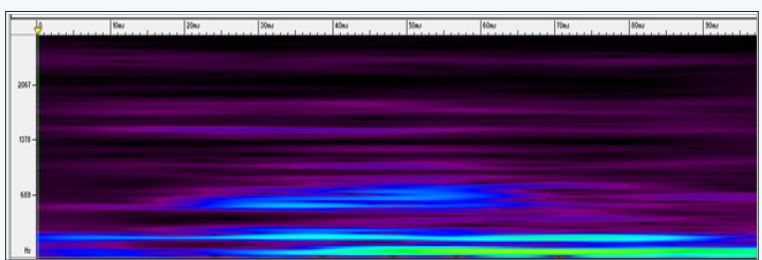

Figure 4: Spectrum of sound click after eating third day male dolphins (Tursiops aduncus).

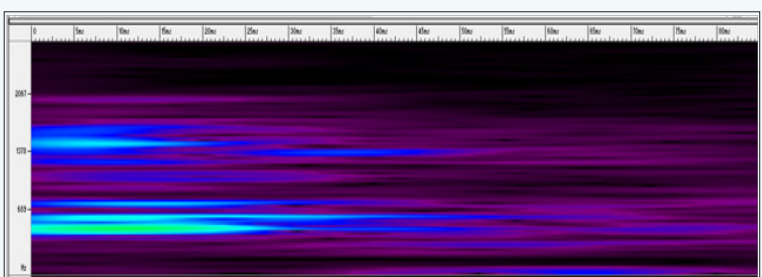

Figure 5: Spectrum of sound click before eating second day male dolphins (Tursiops aduncus)

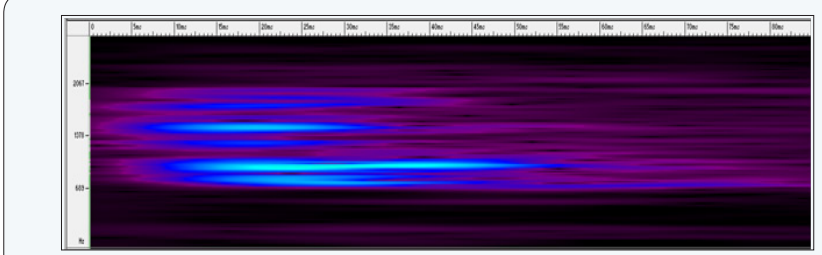

Figure 6: Spectrum of sound click before eating third day male dolphins (Tursiops aduncus).

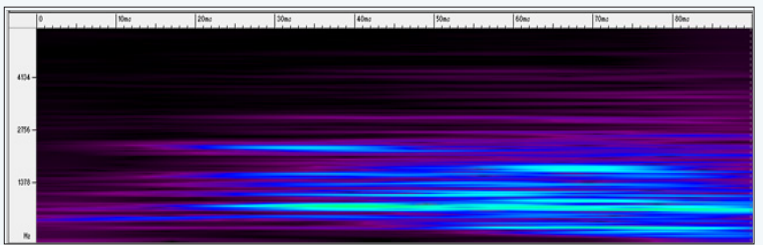

Figure 7: Spectrum of sound click after eating second day male dolphins (Tursiops aduncus).

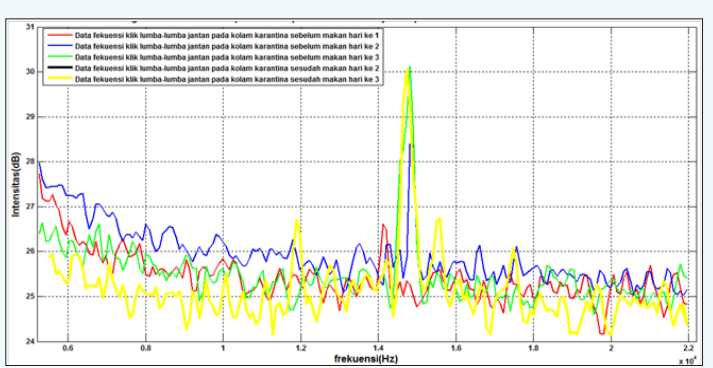

Figure 8: Frequency of relations against intensity before eating day $1,2 \& 3$ and after eating day $2 \& 3$.

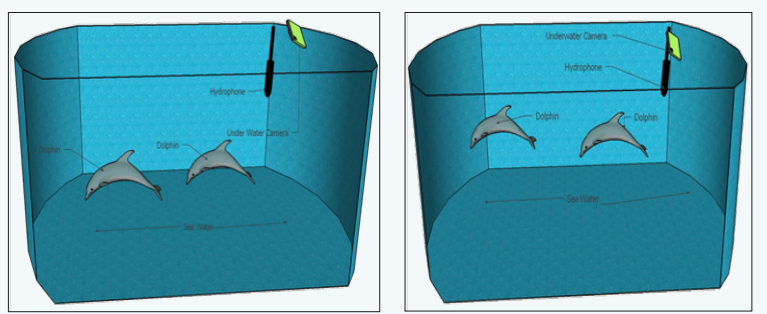

Figure 9: Dolphins position: before eating (a), after eating (b) in quarantine pool.

Result of the spectrum (Figure 3) showed signal fittest present at the data is $20-40 \mathrm{~ms}$, on the outcome of the spectrum (Figure 5) show strongest signal that is at $0-20 \mathrm{~ms}$, on the outcome of the spectrum (Figure 6) show strongest signal that is at $10-30 \mathrm{~ms}$, on the outcome of the spectrum (Figure 7) show strongest signal that is at $30-90 \mathrm{~ms}$, and the outcome of the spectrum (Figure 8) viewed sound or signal shown in the spectrum has been mostly and light in blue very showy that is at sound spectrum after eating day to -2 in seconds to 70 with long sounds namely the $80 \mathrm{~ms}$ with signal of the spectrum the strongest at $40-90 \mathrm{~ms}$. Based with the charts relations frequency to intensity dolphins male at the time before and after eating (Figure 9) with only a maximum frequency $22.000 \mathrm{~Hz}$. On the frequency porpoises porpoises male in quarantine pool before a meal day to 1 in show by a graph red. Data frequency click porpoises porpoises male in the quarantine pool before a meal 
day to 2 blue. Data frequency click dolphins male in quarantine pool before a meal day to 3 colour-coded green. Data frequency click dolphins male in quarantine pool after eating day to 2 in mark with black. Shows the maximum frequency of $22.000 \mathrm{~Hz}$ and with the intensity early $28 \mathrm{~dB}$ and value intensity the end of the $24,82 \mathrm{~dB}$.

Intensity dolphins click 1 having intensity early higher $25,73 \mathrm{~dB}$ and intensity of the low on click 3 namely $24,83 \mathrm{~dB}$. While at the sound of porpoises click 2 have intensity early $24,22 \mathrm{~dB}$. Intensity the end of $23,57 \mathrm{~dB}$, and at the sound of porpoises porpoises click 3 having intensity early 23,83 dB while final score namely $24,77 \mathrm{~dB}$. Based on table 8 after eating day to 3 intensity early dolphins sound in click 1 having 23,59dB and intensity of the end of $24,23 \mathrm{~dB}$, while in click 2 votes dolphins have intensity early namely $24,73 \mathrm{~dB}$ and intensity of the end of the $24,78 \mathrm{~dB}$. In click 3 intensity early namely $24,60 \mathrm{~dB}$ and intensity of the end of the $23,77 \mathrm{~dB}$. Sound click 4 have intensity early namely is $25,63 \mathrm{~dB}$ and intensity of the end of the $25,15 \mathrm{~dB}$.We can see from intensity dolphins click 4 have intensity early higher $25,63 \mathrm{~dB}$ and intensity of the low on click 1 namely $23,59 \mathrm{~dB}$.Value intensity highest look on a chart above is on a range of frequency $14.221-15.100 \mathrm{~Hz}$ In $65-70 \mathrm{~ms}$. Data of frequency click dolphins in quarantine pool after eating day to 3 in give a yellow color. Value intensity highest obtained by the dark line green and yellow that is, before eat day to 3 and after eating day to 3 value intensity by up to $30 \mathrm{~dB}$ in range frequency namely $14.221-15.100 \mathrm{~Hz}$ in range time which is about seconds 65-70.

Value intensity after feeding on click 3 and click 4 having the same value, and this could have been as a received is the same sound that is assuming dolphins sound male same, while compared to a vote before eating. Dolphins preserved in park of safari Indonesia, cisarua bogor the frequency of sounds click with highest intensity of $32,01 \mathrm{~dB}$ with a clicking sound 3 after food in a day to 2 which is found in range frequency namely $14.000-16.000 \mathrm{~Hz}$. The frequency of sounds click dolphins male (Tursiop aduncus) in park of safari is that is at the frequency of $14.000-15.000 \mathrm{~Hz}$. With the intensity lowest of $28,03 \mathrm{~dB}$ and the highest is of $32,01 \mathrm{~dB}$, and according to [29-31] of acoustic signals that is to dolphins namely by range $20 \mathrm{KHz}$. Explore for analysis communication signals of ultrasonic at frequency of the origin of recording of sound whistle and they sound were obtained from two factors that is generally studies species of the dolphins and know who dimilki spectrum pattern.

\section{The behaviour of Dolphins in Quarantine Pool}

The results of recording behavior dolphins with underwater camera looking dolphins dominant lying around on the surface at the time after eating and before eating are at the base and swimming of column sea water in Figure 9. In Figure 9 can be seen dolphins tend to be above the surface in ponds at the time after being given feed and at the time before the given feed dolphins tend to be column at the base and have sea water contamination on an outdoor quarantine pool, showed feeding on the dolphins very influential on behaviour and will result in a weak high sound frequency resulting from the dolphins.

\section{Conclusion}

Sound intensity treatment before and after eating by using the pool the same study have value of the intensity of highest obtained at sound after eating in the second day with their intensity 32,01 dB and value of the lowest intensity of are on before eating in first day. Dolphins tend to be somewhere didasar a pool of sea water on as before eating and dolphins always was sitting on the surface of the pond at the time after eating. The provision of eating treatment very influential on the perceived value of the frequency and the intensity of resulting from male dolphins.

\section{Acknowledgement}

The authors are grateful to the safari park which already allows authors to collect data during the research, and research associates Pratiwi Dwi Wulandari B.Sc in Department Marine Science and Technology, Bogor Agricultural University. The authors also thanks to lecturers and research associates who have participated in this research.

\section{References}

1. Nedwell JR, Parvin SJ (2007) Improvements to Passive Acoustic Monitoring systems. Report No. 565R0810. Subacoustech Ltd. London.

2. Mellinger DK, Stafford KM, Moore SE, Dziak RP, dan Matsumoto H (2007) An Overview of Fixed Passive Acoustic Observation Methods for Cetaceans. Journal of Oceanography 20(4): 36-45.

3. Borowski B, Alexander S, Heui-Seol R, Bunin Barry (2008) Passive acoustic threat detection in estuarine environments. Stevens Institute of Technology: Maritime Security Laboratory. Proc of Society of Photographic Instrumentation Engineers 6945(694513): 1-11.

4. Houser Dorian S, Daniel Crocker E, Reichmuth Colleen, Mulsow Jason, Finneran James J (2007) Auditory Evoked Potentials in Northern Elephant Seals (Mirounga angustirostris). Aquatic Mammals 33(1): 110-121.

5. Erbe C (2002) Hearing abilities of baleen whales (Contractor report \#DRDC Atlantic CR 2002-065). Dartmouth, NS: Defence R\&D Canada -Atlantic, pp. 30.

6. Wartzok D, Ketten DR (1999) Marine mammal sensory systems. In Reynolds JE II \&Rommel SA (Eds.), Biology of marine mammals Washington, DC: Smithsonian Institution Press, pp. 117-175.

7. Bebus SE, Herzing L (2015) Denise Mother-Offspring Signature Whistle Similarity and Patterns of Association in Atlantic Spotted Dolphins (Stenella frontalis) Scinow Publications Ltd. ABC, Animal Behavior and Cognition, 2(1): 71-87.

8. Lubis MZ, Wulandari PD, Harahap MS, Tauhid M, Moron JR (2016) Bioacoustic: Percentage Click Sound of Indo-Pacific Bottlenose Dolphins (Tursiops Aduncus) in Captivity, Indonesia. J Biosens Bioelectron 7(207): 2 .

9. Lubis ZM, Mujahid M, Harahap MS, Tauhid M (2016) Signal Processing: Passive Acoustic in Fisheries and Marine Mammals. J Biosens Bioelectron 7(208): 1-5.

10. Azzolin M, Papale E, Lammers MO, Gannier A, Giacoma C (2013) Geographic variation of whistles of the striped dolphin (Stenella 
coeruleoalba) within the Mediterranean Sea. The Journal of the Acoustical Society of America 134: 694.

11. Papale E, Azzolin M, Cascão I, Gannier A, Lammers MO, et al. (2013) Macro-and micro-geographic variation of short-beaked common dolphin's whistles in the Mediterranean Sea and Atlantic Ocean. Ethology Ecology \& Evolution, p. 1-13.

12. Janik VM, Slater PJ (1998) Context-specific use suggests that bottlenose dolphin signature whistles are cohesion calls, Anim Behav 56(4): 829838.

13. Urick RJ (1975) Principles of Underwater Sound. Kingsport Press, pp. 384 .

14. Simmonds J, MacLennan D (2005) Fisheries Acoustics: Theory and Practice $\left(2^{\text {nd }}\right.$ edn). Blackwell.

15. Popper AN, Hasting MC (2009) The effects of anthropogenic sources of sound on fishes. J Fish Biol. 75(3): 455-489.

16. Urick RJ (1983) Principles of underwater sound. McGraw-Hill, New York, USA.

17. Ramirez D (2012) US Patent Washington, DC: U.S. Patent and Trademark Office 8(189): 797.

18. Wulandari PD, Pujiyati S, Hestirianoto T, Lubis MZ (2016) Bioacoustic Characteristic Click Sound and Behaviour of Male Dolphins Bottle Nose (Tursiops aduncus). J Fisheries Livest Prod 4: 160.

19. Lubis MZ, Pujiyati Sri, Hestirianoto Totok, Wulandari PD (2016) Bioacoustic Characteristics of Whistle Sounds and behaviour of male Indo-Pacific bottlenose dolphins (Tursiops aduncus) in Indonesia. International Journal of Scientific and Research Publications 6(2): 1-7.

20. Lubis MZ, Pujiyati S, Hestirianoto T (2016) Bioacoustic spectral whistle sound and behaviour of male dolphin bottle nose (Tursiops aduncus) at Safari Park Indonesia, Cisarua Bogor. Waste Technology 4(1): 24-30.

21. Brook D, RJ Wynne (1991) Signal Processing: Principples and Applications. Edward Arnold, a division of Hodder and Stoughton Limited, Mill Road, Dunton Green. Great Britain.
22. Krauss TPL Shure JN Little (1995) Signal Processing Toolbox: For Use with Matlab. The Mathworks, Inc.

23. Potts D, Steidl G, Tasche (2001) Fast Fourier transforms for nonequispaced data: A tutorial. In Modern sampling theory. Birkhäuser Boston pp. 247-270.

24. Herzing Denise L (1996) Vocalizations and associated underwater behavior of free-ranging Atlantic spotted dolphins, Stenella frontalis and bottlenose dolphins, Tursiops truncates, Aquatic Mammals. Florida Atlantic University, Biological Sciences, Boca Raton, FL 33431, USA 22(2): 61-79.

25. Lammers, Marc O, Whitlow WL Au (2003) The broadband social acoustic signaling behavior of spinner and spotted dolphins'. Marine Mammal Research Program, Hawaii Institute of Marine Biology, University of Hawaii, (C) 2003 Acoustical Society of America.

26. Jakobsen L, Brinklov S, Surlykke A (2013) Intensity and directionality of bat echolocation signals. Front Physiol 4: 89.

27. Lubis MZ, Pujiyati S (2014) The impact of acclimatization of various salinity to againts mortalitas rate and behaviour fish guppy (poecilia reticulata) as a subtitute for fish bait skipjack (katsuwonuspelamis). Journal teknologi perikanan dan kelautan, 4(2).

28. Pratt M (1998) Better Angling With Simple Science: The White Friars Press. London, England.

29. Winn HE (1991) Acoustic Discrimination By The Road FishWith Comments On Signal System. In Howard E. Winn. Dan Bori J. Olla. (ed) Behavior of Marine AnimalsVertebrates. Plenum Press. New York, USA, 2: 361-381.

30. Lubis MZ (2016) Identifikasi Karakteristik Whistle Dan Tingkah Laku Lumba-Lumba (Tursiops Aduncus) Di Taman Safari Indonesia, Cisarua Bogor(Doctoral dissertation, Bogor Agricultural University (IPB)).

31. Lubis MZ, Pujiyati S, Hestirianoto T, Wulandari PD (2016) Bioacousti c Characteristics of whistle sounds and behaviour of male indo-pacific bottlenos e dolphins (Tursiops aduncus) in Indonesia. International Journal of Scientifi c and Research Publications 6(2): 1-7.

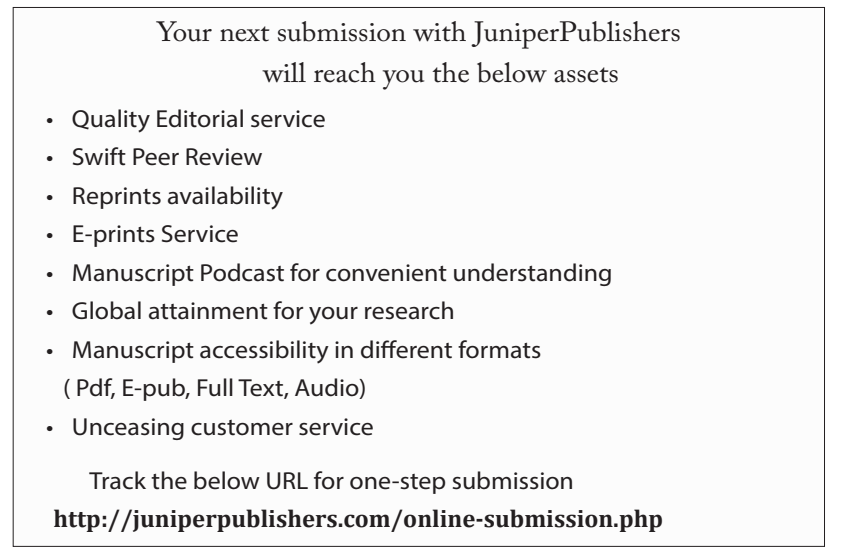

